\title{
SOLARPROP: Charge-sign Dependent Solar Modulation for Everyone
}

\author{
Rolf Kapp: $\left.\right|^{1}$ \\ Bethe Center for Theoretical Physics \\ and \\ Physikalisches Institut der Universität Bonn \\ Nussallee 12, 53115 Bonn, Germany
}

\begin{abstract}
We present SOLARPROP, a tool to compute the influence of charge-sign dependent solar modulation for cosmic ray spectra. SOLARPROP is able to use the output of popular tools like GALPROP or DRAGON and offers the possibility to embed new models for solar modulation. We present some examples for proton, antiproton and positron fluxes in the light of the recent PAMELA and AMS-02 data.
\end{abstract}

\footnotetext{
${ }^{1}$ kappl@th.physik.uni-bonn.de
} 


\section{Introduction}

The study of high energetic cosmic rays is a well established method to constrain possible decay or annihilation of dark matter to standard model particles. Cosmic rays may either have secondary origin e.g. from spallation of primary particles or originate from primary sources like supernova remnants, pulsars or the decay or annihilation of dark matter. The additional cosmic ray flux from dark matter would increase the measured experimental values at earth. To determine the astrophysical background for cosmic rays as well as their possible fraction originating from dark matter, particles have to be propagated through the galaxy from their origin to the solar system. The propagation of charged cosmic rays is well studied in the literature [1 5] and despite the still large uncertainty, an enormous progress has been made during the last years. As an outcome freely available tools like GALPROP [2, 3] and DRAGON 4.5 exist, which simulate the propagation of cosmic rays through the galaxy.

Once, the cosmic rays have reached the solar system, they are further influenced by the solar wind and the magnetic field of the sun [6]. This effect is called solar modulation. It can be approximately taken into account with the so-called force-field method [7]. This approximation is independent of the charge of the cosmic ray particles. The strength of solar modulation depends on the solar activity and thus on the 22 year solar cycle. High solar activity results in a stronger effect and thus a lower cosmic ray flux, whereas lower solar activity results in a smaller effect and a higher cosmic ray flux at the top of the earth's atmosphere. The study of solar modulation is a field of intensive research and many models which are able to provide a better estimate than the force-field approximation for the influence of the heliosphere on cosmic ray transport have been proposed (see [8,9] for recent effective approaches based on the force-field approximation).

Solar modulation can be well described by a Fokker-Planck equation [6]. It has become popular to rewrite this equation to a set of stochastic differential equations (see e.g. [10 16] ) which can be solved with Monte Carlo methods. SOLARPROP [17] is a simple Monte Carlo tool which is flexible enough that arbitrary models for solar modulation in the heliosphere can be embedded. It includes several simple models which show the effect of a more realistic model for solar modulation. We outline that charge-sign dependent models nicely fit the experimental data and are an advantage to the force-field approximation. The force-field approximation is based on one free parameter, the so-called Fisk potential $\phi$. It serves as a free fit parameter, adjusted a posteriori to model the effect of solar modulation. In contrast, the models in SOLARPROP are based on experimental values of the solar magnetic field. This makes it possible to model solar modulation without a fit parameter but in a predictive framework. For any given date in the past, different experimental values for the solar activity like the tilt angle of the heliospheric current sheet are used to model the effect of solar modulation in a predictive way.

A better understanding of solar modulation is especially interesting for antiprotons as their flux peaks around a kinetic energy of a few $\mathrm{GeV}$, an energy range where the effect of solar modulation is very strong. Due to the low astrophysical background, antiprotons have been used to constrain dark matter models (see e.g. [18 for an analysis of the BESS-Polar II data). A lot of balloon based data are available at a few $\mathrm{GeV}$ and a proper treatment of solar modulation can help to decrease the uncertainties on indirect dark matter detection.

SOLARPROP is easy to use and easy to extend with new custom models. The program is able to deal with input in FITS format from GALPROP and DRAGON, as well as with input from text files. The output is provided in the same formats. This makes it possible to include 
SOLARPROP in the chain of cosmic ray propagation tools. A realistic treatment of cosmic ray propagation from the production in the galaxy to the detection at the top of the earth's atmosphere is thus possible.

\section{Physical background}

The transport equation for cosmic rays in the heliosphere is given by 6, 19

$$
\frac{\partial f}{\partial t}=\nabla \cdot(\kappa \cdot \nabla f)-\left(\mathbf{V}+\mathbf{V}_{D}\right) \cdot \nabla f+\frac{1}{3}(\nabla \cdot \mathbf{V}) \frac{\partial f}{\partial \ln p}
$$

$f$ is the particle phase space distribution function, $\kappa$ the diffusion tensor, $\mathbf{V}$ the solar wind velocity, $\mathbf{V}_{D}$ the particle drift velocity in the heliospheric magnetic field and $p$ the particle momentum. This Fokker-Planck equation can be rewritten to an equivalent set of stochastic differential equations (SDEs) [20, 21]. The idea has been used by many authors $[10-16$ to solve the propagation of cosmic rays in the heliosphere with Monte Carlo techniques.

The SDEs can be written as

$$
d x_{i}=A_{i}\left(x_{i}\right) d t+\sum_{j} B_{i j}\left(x_{i}\right) d W_{j}
$$

where $x_{i}$ are some coordinates of pseudo-particles, $t$ is the time, $A_{i}$ is a drift and $B_{i j}$ a diffusion term. $W_{j}$ is a Wiener process which can be related to a Gaussian distribution $d w_{j}$ with mean zero and standard deviation of one $N(0,1)$ by $d W_{j}=\sqrt{d t} d w_{j}$. The desired particle phase space distribution $f$ is now obtained by solving the SDEs for a large sample of pseudo-particles. The SDEs for a simple one dimensional example are given by 10

$$
\begin{aligned}
\Delta r & =\left(-V+\frac{2 \kappa_{r r}}{r}\right) \Delta t+\sqrt{2 \kappa_{r r} \Delta t} d w_{r}, \\
\Delta T & =\frac{2 V}{3 r} \frac{T^{2}+2 T m}{T+m} \Delta t
\end{aligned}
$$

with radial coordinate $r$, particle mass $m$ and kinetic energy $T . V$ labels a constant solar wind and $\kappa_{r r}$ is the energy dependent diffusion constant. The initial condition at the boundary of the heliosphere which we typically set to $r_{\text {Boundary }}=100 \mathrm{AU}$ for $t=0$ is the local interstellar flux. One can solve the SDEs in the so-called forward time approach. One starts with initial discrete kinetic energies $T_{i}$ and simulates for small time steps $\Delta t$ the evolution of $T_{i}$ according to the SDE until either some large finite time or the position of the earth at $r_{\text {Earth }}=1 \mathrm{AU}$ is reached. If the earth is reached, the final value $T=T_{i}$ is recorded. Doing this for many pseudo-particles results in a continuous energy distribution for the same initial energy $T_{i}$ of pseudo-particles. After normalization to one initial pseudo-particle this gives us a kind of Green's function $G\left(T, T_{i}\right)$ which indicates for every initial energy $T_{i}$ of some particle the probability to have kinetic energy $T$ after reaching the earth (see e.g. 14 for a more detailed discussion).

SOLARPROP solves the SDEs in a different, so-called backward approach. Pseudo-particles start at the earth and are simulated backward in time until they reach the heliosphere. It has been shown that this approach is usually faster then the forward approach [21, 22]. To incorporate the boundary condition, we weight the local interstellar flux $\Phi^{\mathrm{LIS}}(T)$ with the 
Green's function to obtain the flux at the top of the atmosphere $\Phi^{\mathrm{TOA}}(T)$. The flux is related to the phase space distribution by

$$
\Phi^{\mathrm{LIS}}(T)=p^{2} f^{\mathrm{LIS}}(T)=\left(T^{2}+2 T m\right) f^{\mathrm{LIS}}(T)
$$

and we get

$$
f^{\mathrm{TOA}}(T)=\sum_{i} G\left(T, T_{i}\right) f^{\mathrm{LIS}}\left(T_{i}\right), \quad \Phi^{\mathrm{TOA}}(T)=p^{2} f^{\mathrm{TOA}}(T) .
$$

As the propagation depends on the mass of the cosmic rays, SOLARPROP calculates a Green's function for every given cosmic ray species independently. The main physical input is the definition of the solar magnetic field, the diffusion tensor and the treatment of the drift especially along the heliospheric current sheet (HCS). SOLARPROP has several different builtin models and can also easily extended by the user.

\section{Installation}

SOLARPROP is freely available for download under [17]. It needs the two packages cfitsio and CCfits to handle the FITS format. The packages can be downloaded and installed from

http://heasarc.gsfc.nasa.gov/fitsio/

and

http://heasarc.gsfc.nasa.gov/fitsio/ccfits/

Probably one has to tell SOLARPROP where these packages are installed on the system. After successful installation of the libraries, the following steps are necessary to install SOLARPROP. First, the code has to be extracted in a given favorite directory

tar -xvf solarprop.tar.gz

Let us assume the packages cfitsio and CCfits are installed in /usr/local/cfitsio and /usr/local/CCfits. There are two ways to tell SOLARPROP where the packages are. Either through environment variables or during the configure step of the installation.

1. Using environment variables the steps are

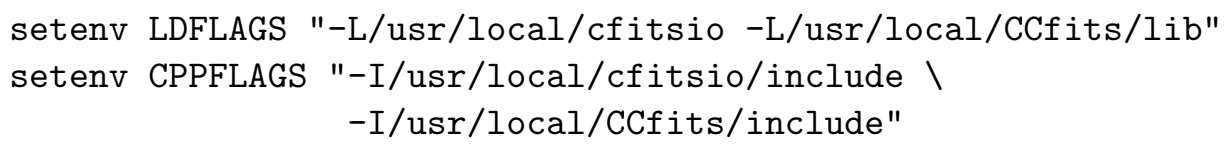

for csh based systems or

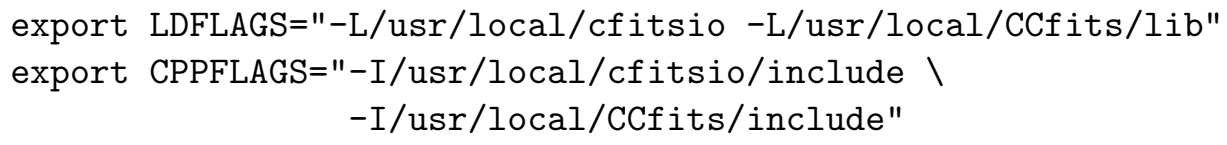

on bash like systems. Finally one has to run

. / configure

2. One can instead also use configure options and do 


$$
\begin{aligned}
. / \text { configure } & \text {--with-CCfits=/usr/local/CCfits } \\
& \text {--with-cfitsio=/usr/local/cfitsio }
\end{aligned}
$$

To install SOLARPROP in an individual directory one can use the --prefix option like

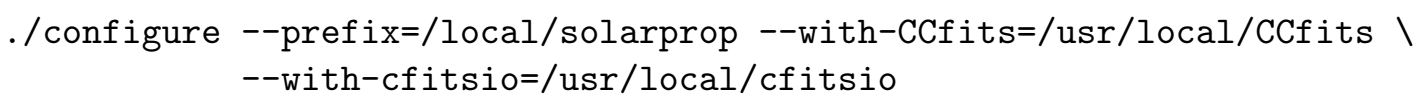

After that the next steps are

make

make install

to install SOLARPROP. The executable should be available in the subdirectory ./bin. The simple call ./bin/SOLARPROP -v should result in This is SOLARPROP version 1.0 if the installation is successful.

\section{Usage of SOLARPROP}

After successful installation SOLARPROP can be used from the command line. There is no interactive user interface, everything is controlled with command line arguments and a control file. The program modulates a given or built in local interstellar flux and writes the computed top of the atmosphere (TOA) flux to a file. The physical model responsible for solar modulation is selected via a parameter in the control file. The local interstellar flux can be given either by a text or FITS file or by the parametrization of the physical model. For an easy start we discuss different simple use cases.

\subsection{Use case 1: Input from GALPROP}

GALPROP stores the computed local interstellar flux of all defined cosmic ray species in a FITS file (see section 4.5 or [23] for a description of the format). This file can directly be used as input for SOLARPROP.

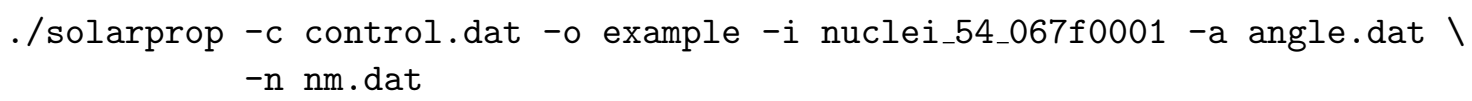

The output of SOLARPROP is determined by the keywords in control.dat. An example for a control file is given below:

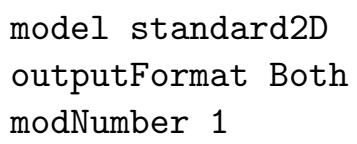

If this control file is used, SOLARPROP creates two output files, example. dat with text output and example.fit with the same data as FITS output. The keyword modNumber has the value 1, thus SOLARPROP calculates the modulation of the first cosmic ray species in the input FITS file from GALPROP. All possible control file options are displayed in table 2 , 3 and 4. 


\subsection{Use case 2: Input from DRAGON}

DRAGON stores the computed local interstellar flux of the different cosmic ray species in a three dimensional FITS file, in a two dimensional FITS file and in a text file. SOLARPROP is able to handle the two dimensional FITS file. A brief description of the format can be found in section 4.5. A simple program call would be

./solarprop -c control.dat -o example -i run_2D_spectrum.fits -n nm.dat

The output of SOLARPROP is determined by the keywords in control.dat. Consider the following control file:

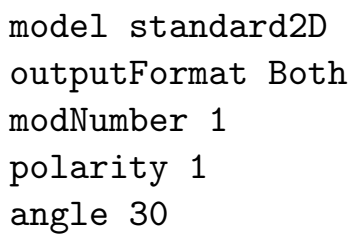

SOLARPROP creates two output files, example.dat with text output and example.fit with the same data as FITS output. The keyword modNumber has the value 1, thus SOLARPROP calculates the modulation of the first cosmic ray species in the FITS file from DRAGON. The solar polarity and the tilt angle of the HCS are manually provided in the control file with the keywords polarity and angle. All possible control file options are displayed in table 2 , 3 and 4 .

\subsection{Use case 3: Input from text file}

SOLARPROP can also handle input from text files. In this case the kinetic energy of the nuclei has to be given in $\mathrm{GeV}$ in the first column, the flux is expected in the second column. The mass and charge of the cosmic ray nuclei can be provided through the mass and charge keywords in the control file.

./solarprop -c control.dat -o example -i textInput.dat -a angle.dat -n nm.dat

The output of SOLARPROP is determined by the keywords in control.dat. A possible control file is shown below:

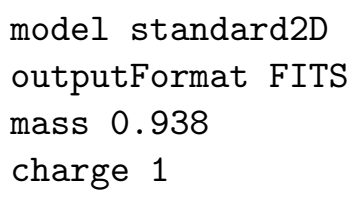

Here outputFormat is given as FITS, thus SOLARPROP creates one output file example.fit with FITS output. The FITS file consists of two columns. The first one with the kinetic energy and the second one with the modulated flux. The keywords mass and charge specify the cosmic ray species to be protons.

\subsection{General use of SOLARPROP}

All possible command line arguments of SOLARPROP are given in table 1. Everything else is controlled by the control file. Every line of the control file is interpreted as key value pair. The first word is a keyword, after a blank, an option should be provided. All keywords are listed in table 2 and 3 . There exists a default behavior of SOLARPROP for each keyword, for 


\begin{tabular}{|c|c|}
\hline Option & escription and argument \\
\hline -c, --control & rgument is the file name where the control file is located. \\
\hline$-a,--a n g l e$ & $\begin{array}{l}\text { Argument is the file name where the tilt angle file is located. The } \\
\text { format should match the definitions from }[24] \text {. }\end{array}$ \\
\hline -o, --output & $\begin{array}{l}\text { Argument is the file name where the output should be stored. The } \\
\text { file ending is added automatically depending on the option provided } \\
\text { in the control file. }\end{array}$ \\
\hline$-i,--i n p u t$ & $\begin{array}{l}\text { Argument is the file name where the input file is located. SOLARPROP } \\
\text { automatically determines if the input is in FITS or Text format. }\end{array}$ \\
\hline$-\mathrm{n},--\mathrm{ne}$ & $\begin{array}{l}\text { Argument is the file name where the file with the neutron monitor } \\
\text { data is located. The file should be in the format from [25. }\end{array}$ \\
\hline$-\mathrm{v},--\mathrm{v}$ & $\begin{array}{l}\text { No argument necessary. If this option is chosen SOLARPROP only dis- } \\
\text { plays the version number. }\end{array}$ \\
\hline
\end{tabular}

Table 1: Command line options.

example in case no option is given, or the option is ill-defined. The possible options are given in table 4 .

A general program call is:

. solarprop -c control.dat -a angle.dat -n nm.dat -o example -i input.fits

The two files angle.dat and $\mathrm{nm}$.dat store the tilt angle and neutron monitor data which are necessary for the model standard2D (see the discussion in section 5.2). If another model is used, these files are not necessary. If one wants to adjust the result from SOLARPROP, e.g. to find a better agreement with a data set, the options kappaScaling and BfieldScaling can be used. The first one parametrizes the normalization of the diffusion, whereas the second one parametrizes the strength of the solar magnetic field. A larger value for kappaScaling e.g. 1.5 makes the diffusion more efficient and results in a higher top of the atmosphere flux.

If the option phi is given in the control file, SOLARPROP also calculates the result for the force-field approximation. This result is stored in a file with the name exampleForce.dat or exampleForce.fit depending on the value for outputFormat.

\subsection{Input and output of SOLARPROP}

The local interstellar input flux for SOLARPROP can be provided through the command line option $-i$ in three different ways.

1. If no input file is provided, SOLARPROP tries to find a reference local interstellar flux provided by the chosen model. If that is not successful, a warning is displayed and a vanishing local interstellar flux is assumed. In that case the output is useless.

2. The local interstellar flux can be imported from a text file. The first column is assumed to contain the kinetic energy in $\mathrm{GeV}$ and the second column the local interstellar flux in $\mathrm{m}^{-2} \mathrm{sr}^{-1} \mathrm{~s}^{-1} \mathrm{GeV}^{-1}$. The mass and charge of the cosmic ray species have to be given in the control file.

3. If a FITS file is given, SOLARPROP checks automatically if the file is in GALPROP or DRAGON like format. Other formats are not supported. In case a modNumber is given, 


\begin{tabular}{ll} 
Option & Description \\
\hline model & Defines the physical model which should be used for solar modulation. \\
phi & Default is ref1. \\
Force-field value in GV if provided. Result is stored in separate output. \\
Dofnumber
\end{tabular}

Table 2: Control file options (Part 1).

only the desired cosmic ray species is modulated. If no modNumber is found, SOLARPROP modulates all cosmic ray species found in the import file.

The output of SOLARPROP is stored in files which names are provided through the option -o. The output depends on the keywords modNumber and outputFormat and on the format of the input. We can distinguish between two cases.

1. If the input is from a text file or the local interstellar flux is from an internal reference model, independent of the modNumber only one cosmic ray species is modulated as only one is provided. In the case of text file output, in the first column the kinetic energy in $\mathrm{GeV}$ is written. In the second column the top of the atmosphere flux is provided in units of $\mathrm{m}^{-2} \mathrm{sr}^{-1} \mathrm{~s}^{-1} \mathrm{GeV}^{-1}$ (if index is 0 ). The energy bins are always the same as the input ones from the local interstellar flux.

In case of FITS output, two keywords, namely mass and charge to store the cosmic ray properties are added to the pHDU. The first column of the pHDU consists of the kinetic energy in $\mathrm{GeV}$ and the second column stores the calculated flux in $\mathrm{m}^{-2} \mathrm{sr}^{-1} \mathrm{~s}^{-1} \mathrm{GeV}^{-1}$ (if index is 0 ). 


\begin{tabular}{|c|c|}
\hline Option & escription \\
\hline polarity & $\begin{array}{l}\text { Defines solar polarity manually. Default is } 1 \text {. Option is only used if } \\
\text { the command line option -a, --angle is not used. }\end{array}$ \\
\hline angle & $\begin{array}{l}\text { Defines the tilt angle manually. Default is } 0 \text {. Option is only used if } \\
\text { the command line option -a, --angle is not used. }\end{array}$ \\
\hline outputFormat & Defines the format for the output file. Default is Text. \\
\hline kappaScaling & $\begin{array}{l}\text { Normalization of the diffusion tensor } \kappa \text { can be manually adjusted. } \\
\text { Default is } 1 .\end{array}$ \\
\hline BfieldScaling & $\begin{array}{l}\text { Normalization of the magnetic field } B \text { can be manually adjusted. De- } \\
\text { fault is } 1 \text {. }\end{array}$ \\
\hline index & $\begin{array}{l}\text { Spectral index for output rescaling. Output flux is rescaled with } \\
T^{\text {index }} \text {. Default is no rescaling } 0 .\end{array}$ \\
\hline$d t$ & $\begin{array}{l}\text { Used time step } \Delta t \text { can be adjusted manually. Be careful with this } \\
\text { option. A too large time step increases the speed, but results in wrong } \\
\text { results. A too small time step results in a very high computing time. }\end{array}$ \\
\hline extraBins & $\begin{array}{l}\text { Number of extra bins which should be added between the given local } \\
\text { interstellar spectrum data points. Computed bins are only added if } \\
\text { the given data points from the input file are not dense enough. Default } \\
\text { is } 5 \text {. }\end{array}$ \\
\hline total & $\begin{array}{l}\text { Number of pseudo-particles which are modulated for every energy bin. } \\
\text { Default is } 1000 \text {. }\end{array}$ \\
\hline nmValue & $\begin{array}{l}\text { Value for the diffusion tensor normalization in the standard2D model. } \\
\text { Default is } 500 \text {. Option is only used if the model standard2D is selected } \\
\text { and command line option }-\mathrm{n},-- \text { neutron is not used. }\end{array}$ \\
\hline
\end{tabular}

Table 3: Control file options (Part 2).

2. If the input is from a FITS file, depending on the value of the keyword modNumber one or all cosmic ray species in the file are modulated. All species are present in the output. If the species is not modulated the local interstellar flux is added to the output. In the case of text output, the file consists of one column with the kinetic energy in $\mathrm{GeV}$ and additional columns, one for the flux of each cosmic ray species in $\mathrm{m}^{-2} \mathrm{sr}^{-1} \mathrm{~s}^{-1} \mathrm{GeV}^{-1}$ (if index is 0 ).

For FITS output, SOLARPROP uses the same format as given by the input. That means, if the input is in GALPROP like format the fluxes are stored, each in a separate column in the pHDU, whereas for a DRAGON like format every flux is stored in its own HDU. Please note that the unit of the stored fluxes in both cases is changed to the SOLARPROP standard $\mathrm{m}^{-2} \mathrm{sr}^{-1} \mathrm{~s}^{-1} \mathrm{GeV}^{-1}$ !

\subsection{Inclusion of custom models}

SOLARPROP can be easily extended by the user. The main classes for the physics of particle propagation are the interface (abstract base class) Iparticle.h, the derived abstract class particle.cc and its concrete implementations like standard2D.cc. If one wants to include an own model, it should inherit from particle.cc. Of course, all virtual methods of particle.cc have to be implemented and a constructor has to be added. The method void calculate() manages the computation of all quantities which are needed several times in 


\begin{tabular}{ll} 
Keyword & Allowed parameters \\
\hline model & ref, ref2, ref3, ref4, standard2D or custom. \\
phi & Every numerical value. Unit is GV. \\
modNumber & $\begin{array}{l}\text { Integer value. Value should not be higher than the number of cosmic } \\
\text { ray species in the input FITS file. }\end{array}$ \\
mass & Every numerical value. Unit is GeV. \\
charge & Integer value. \\
tiltModel & L or R. \\
year & Four digit integer value. \\
month & One or two digit integer value. \\
yearEnd & Four digit integer value. \\
monthEnd & One or two digit integer value. \\
polarity & 1 or -1. \\
angle & Every numerical value between 0 and 90. Unit is degree. \\
outputFormat & FITS, Text or Both. \\
kappaScaling & Every numerical value. \\
BfieldScaling & Every numerical value. \\
index & Every numerical value. \\
dt & Integer value. \\
extraBins & Integer value. \\
total & Integer value. \\
nmValue & Every numerical value.
\end{tabular}

Table 4: Viable values for the keywords in the control file as described in table 2 and 3 .

a propagation step. This avoids unnecessary double computations. A basic implementation can already be found in particle.cc.

SOLARPROP already offers two files for the concrete implementation of a custom model, custom.h and custom.cc. The implemented model can be called from the control file with the name custom as described for the validation in import.h.

\section{Examples and validation}

\subsection{Validation}

We validate SOLARPROP against several models available in the literature. This approach has also been used previously to test Monte Carlo based solar modulation calculations [14]. A comparison of the result from SOLARPROP with the result of [10] and [26] is shown in figure 1. In the left panel a one dimensional model with plain diffusion and a diffusion coefficient $\kappa=5 \cdot 10^{22} \mathrm{~cm}^{2} \mathrm{~s}^{-1} \mathrm{GV}^{-1}$ is used (matches figure 4 in $[10]$ ). The model is implemented for reference in SOLARPROP as model ref1. The corresponding SDEs can be found in appendix A.1.1. The control file to reproduce this result is very simple:

model ref 1

mass 0.938

charge 1

The corresponding program call is: 


\section{./solarprop -c control.dat -o example}

In the right panel a two dimensional model without tilt angle dependence (flat HCS) is used. The parameters match the ones in table 1 and figure 2 of [26]. The model can be used in SOLARPROP with parameter ref 2 and the SDEs can be found in appendix A.2.1. For negative polarity $A<0$ the control file to reproduce the figure is:

model ref 2

mass 0.938

charge 1

polarity -1

A comparison of more sophisticated models is displayed in figure 2, In the left panel a two
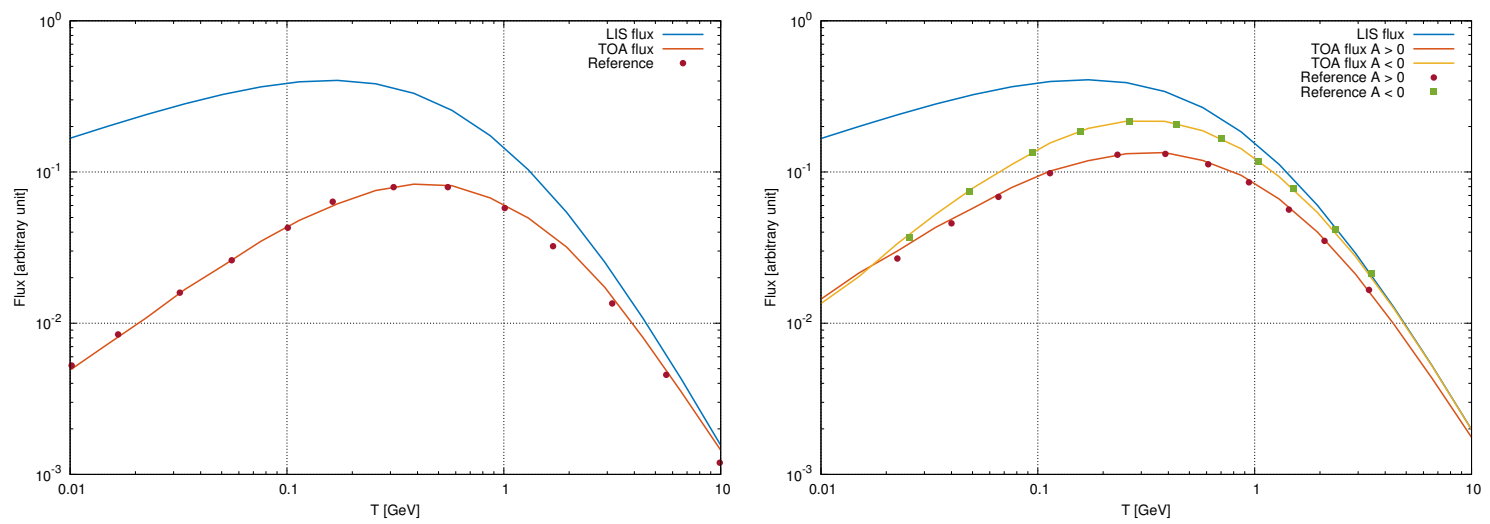

Figure 1: Validation of SOLARPROP with the reference model ref1 from [10] (left panel) and the model ref2 from 26 (right panel).

dimensional model with a possibility to model a wavy HCS is used [27]. The plot displays a comparison between SOLARPROP and the result from figure 6 of [27]. The SDEs can be found in appendix A.2.2. The corresponding control file for positive polarity $A>0$ is:

model ref3

mass 0.938

charge 1

polarity 1

In the right panel the result of figure 5 from 28 is reproduced for two different values of the tilt angle $\alpha$. The model is also two dimensional and able to describe a wavy HCS. As can be seen in appendix A.2.3 the drifts are described in a different way than in 27. The following options have been used to produce the result for the case $A<0$ and $\alpha=30^{\circ}$.

model ref 4

mass 0.938

charge 1

polarity -1

angle 30

The models of figure 2 are available in SOLARPROP under the name ref 3 and ref 4 respectively. The results from figure 1 and figure 2 nicely show that our Monte Carlo approach is viable 

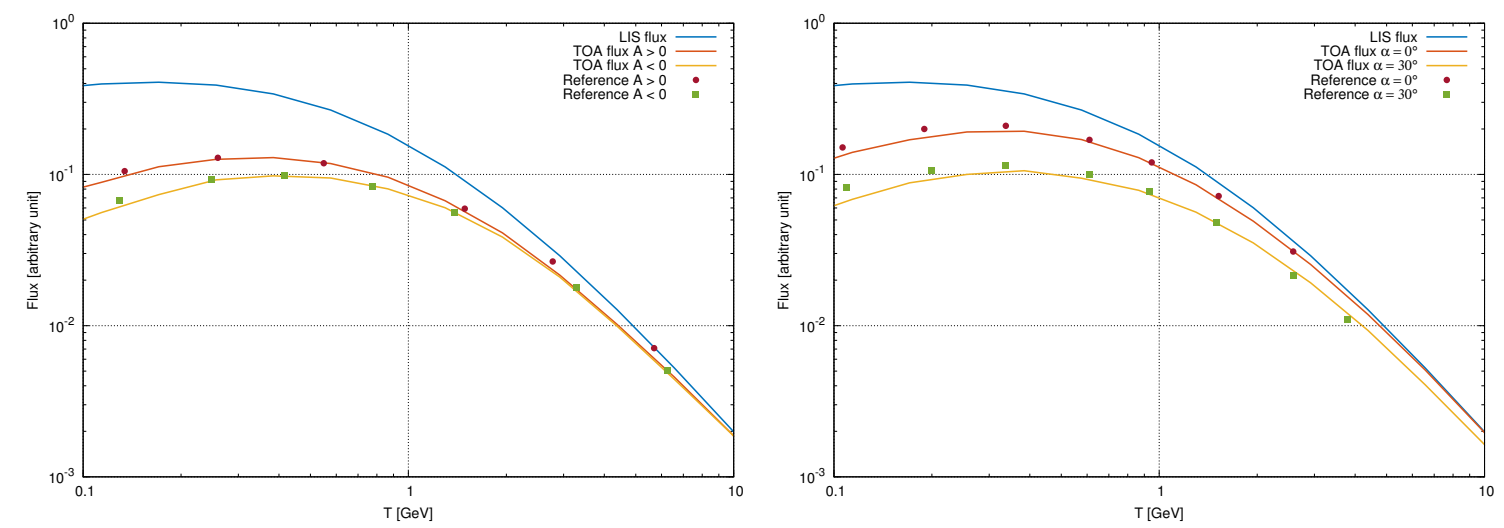

Figure 2: Validation of SOLARPROP with the reference model ref3 from 27] (left panel) and the model ref 4 from 28 (right panel).

and different models of the heliosphere can be easily implemented in SOLARPROP.

\section{$5.2 \quad$ Examples}

We want to introduce in this section another model which is implemented in SOLARPROP and show its result with different data sets. This more sophisticated model, able to describe recent cosmic ray data is implemented in SOLARPROP under the name standard2D. The model is still very simple, but has several time dependent parameters to accurately describe recent data. A more complicated model, e.g. a three dimensional one, can easily added by the user or will maybe be part of the next version of SOLARPROP. A challenging data set for cosmic ray protons released by the PAMELA experiment [29] is displayed in figure 3 . The proton flux increases with time which indicates a decreasing solar activity. This data set has been successfully described by solar modulation models [16, 30. These studies have shown that a simple model, where only one parameter (e.g. the tilt angle) is time dependent is insufficient to describe the data. This excludes all our discussed reference models. In table 5 several measured solar activity dependent parameters are displayed. To accurately describe

\begin{tabular}{ccccc} 
Time & $\alpha_{L}$ & $\alpha_{R}$ & SSN & NM count rate (Newark) \\
\hline 11.2006 & 29.5 & 11.25 & 12.6 & 3539 \\
12.2007 & 31.267 & 15.8 & 4.9 & 3591 \\
11.2008 & 26 & 9.85 & 1.7 & 3678 \\
12.2009 & 28.3 & 13.55 & 8.3 & 3732
\end{tabular}

Table 5: Different solar activity dependent parameters as measured by 24, 31 33 for a time of negative solar polarity $A<0$. The increasing NM count rate indicates a decreasing solar activity.

experimental data a tilt angle and sunspot number (SSN) dependent model was used in [12]. In [16,30 many more parameters, like the overall magnetic field strength have been adjusted. We use a tilt angle dependent model and vary in addition the normalization of the diffusion tensor $\kappa_{0}$, taking into account a more or less effective diffusion.

The variation of $\kappa_{0}$ is based on the calculations of 34,35$]$ which average different neutron monitor (NM) count rate data. In [34,35] a force-field potential $\phi_{\text {Usoskin }}$ was determined from 


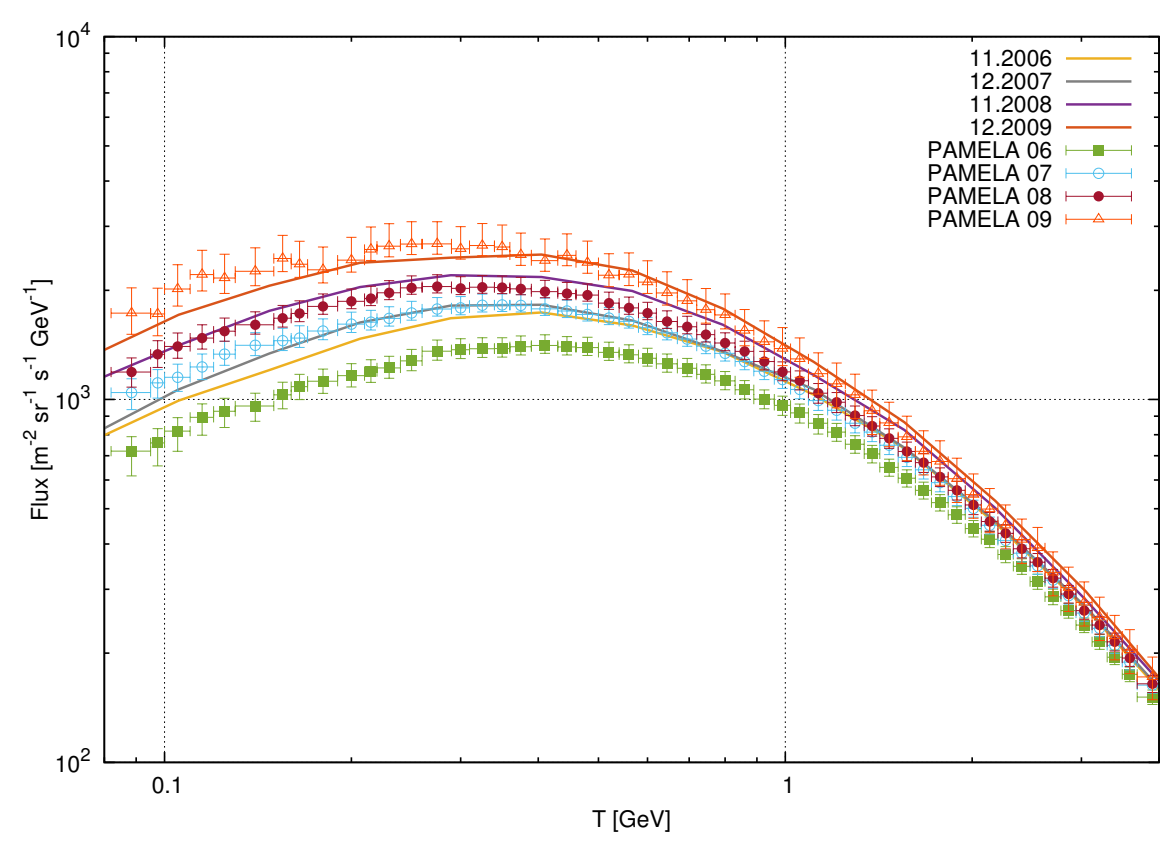

Figure 3: Data for cosmic ray protons at different solar activities measured by PAMELA [29]. The result from SOLARPROP with the model standard2D is displayed as solid lines. The result is in good agreement, except for the data from 2006.

the neutron monitor data to describe charge-sign independent solar modulation. We relate the diffusion tensor normalization $\kappa_{0}$ to this quantity $\phi_{\text {Usoskin. By not directly using the }}$ neutron monitor data we avoid to redo the average procedure, already performed by [34, 35]. We use the simple relation

$$
\kappa_{0}=\tilde{\kappa_{0}} \begin{cases}\frac{137}{\phi_{\text {Usoskin }}}-0.061 & , q A<0 \\ \frac{7}{100} \frac{137}{\phi_{\text {Usoskin }}}-0.061 & , q A>0\end{cases}
$$

to model a solar activity dependent diffusion tensor normalization. $\tilde{\kappa_{0}}$ is just the usual diffusion constant. The formula depends on the polarity of the solar cycle $A$ and the charge of the cosmic ray species $q$ to take into account that drift effects have not been considered in [34, 35. This idea is also motivated by the approach in 12$]$ where the normalization of the diffusion tensor is polarity and charge dependent. The detailed implementation of the drift effects is described in appendix A.2.4. To reproduce the results in figure 3 e.g. for the date 11.2007 the control file is:

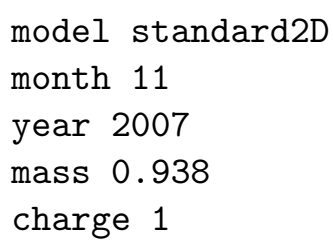

Except for the data from 2006 the model standard2D is in good agreement with the data. To describe all four data sets a more complicated model is necessary.

We want to point out that the concrete form of a successful model for solar modulation strongly depends on the local interstellar flux. Obviously, a different shape of the diffusion 
tensor or a different approach to model the drift effects is possible if the local interstellar flux is adjusted. As the Voyager 1 spacecraft has recently passed the heliosphere and entered the heliopause [36], the local interstellar flux for some cosmic ray species is now measured directly for the first time. We show this data in figure 4 together with different local interstellar proton fluxes. We take the local interstellar proton flux from [37] as also used in [12,34,35]

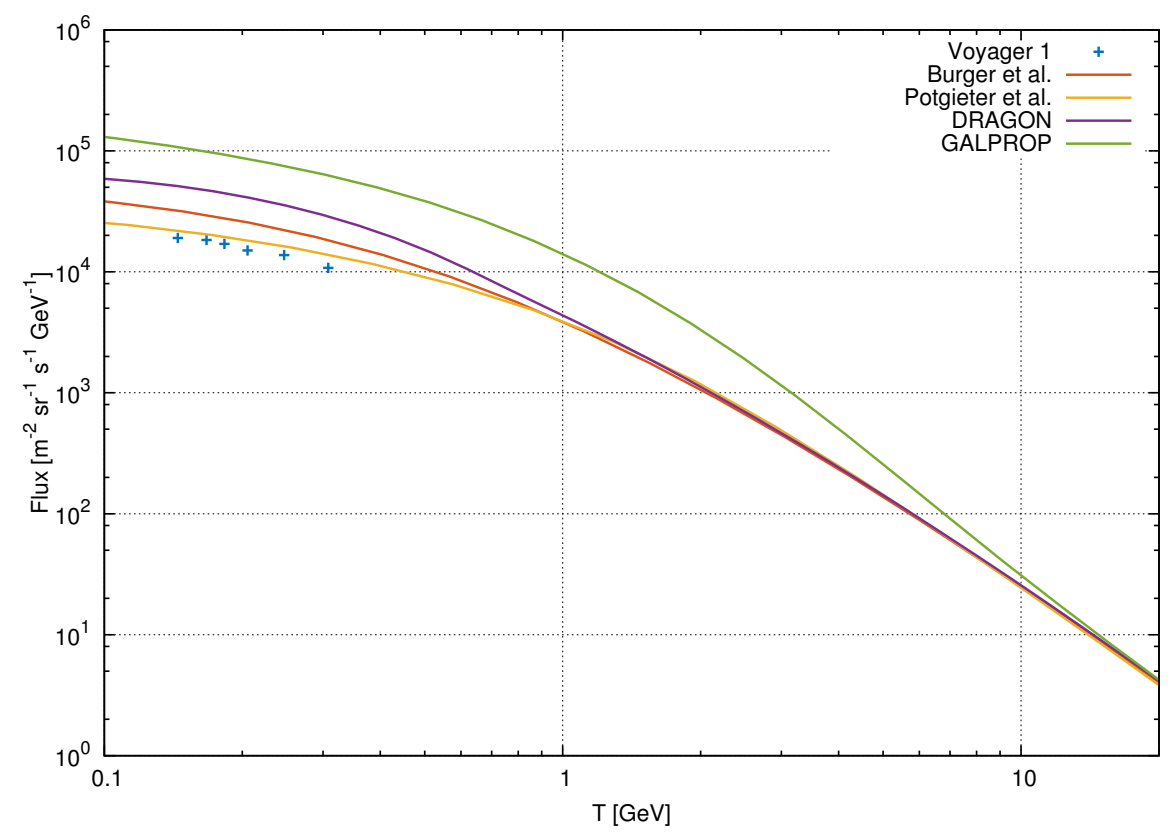

Figure 4: Voyager 1 measurements for protons at $122 \mathrm{AU}[36]$ and different popular local interstellar proton fluxes [16, 37 including results from GALPROP and DRAGON. The fluxes from GALPROP and DRAGON are of course model dependent.

(notice the comment in 34 about the different forms of the equation). As figure 4 indicates, the flux from [16] seems to be more appropriate. Because we use results from [34, 35, we restrict ourselves to the proton flux from [37]. This local interstellar flux is used for the result displayed in figure 3 .

In figure 5 the result for cosmic ray modulation with the standard2D model of SOLARPROP and data from the BESS and BESS-Polar experiments [38 43] are displayed. The local interstellar flux for antiprotons is determined with recent results from the literature. The galactic propagation setup described in [44] together with the recently updated antiproton production cross sections 45] and the proton flux from [37] is used. Emphasizing that the model uses no fit parameters it describes the data very well. The only input is the time when the experiment took place. The result slightly overshoots the data from the BESS-Polar II experiment which took data in December 2007. As there is a deviation between the PAMELA proton data from the same time period and the BESS-Polar II data we do not worry about this result.

One can also describe leptons as can seen in figure 6. This data (especially the positron fraction) has also been discussed in [49] as indication for charge-sign dependent solar modulation. The local interstellar positron flux seems to include an exotic primary contribution at higher energies, known as the positron excess (see e.g. 50 for a recent discussion of the possible primary positron contribution).

For the parametrization we use the secondary positron flux from DRAGON $\Phi_{\text {sec, DRAGoN }}^{\mathrm{LIS}}(T)$ 

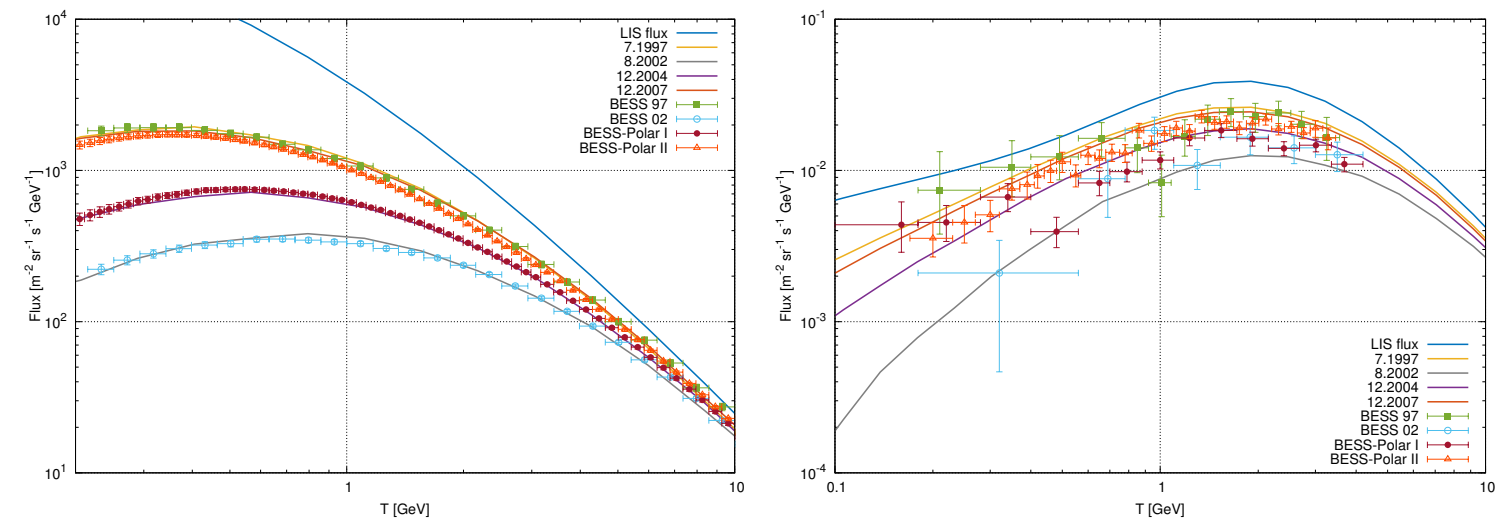

Figure 5: Data for cosmic ray protons and antiprotons at different solar activities measured by BESS 97, BESS 2002 [38 40] and BESS-Polar I, BESS-Polar II 41 43].

and add a power law contribution $\Phi_{\text {prim }}^{\mathrm{LIS}}(T)=3.7 \cdot T^{-2.7}$ to model the primary contribution. This flux choice $\Phi_{e^{+}}^{\mathrm{LIS}}(T)=\Phi_{\text {Sec, DRAGON }}^{\mathrm{LIS}}(T)+\Phi_{\text {prim }}^{\mathrm{LIS}}(T)$ is in agreement with the high energy data by PAMELA [47] and AMS-02 [48]. The result for solar modulation with this local interstellar flux and the model standard2D is in poor agreement with the data. This shows that we need a refined model to describe also solar modulation for leptons in a reasonable way or the local interstellar flux for positrons is different. It has recently been shown that more sophisticated models [51] can well describe new electron data by the PAMELA experiment [52]. The inclusion of such models which seem to be able to describe solar modulation for leptons in SOLARPROP is left for future work. To reproduce e.g. the theoretical estimate for the positron flux of the AMS-02 experiment displayed in figure 6 the control file is:

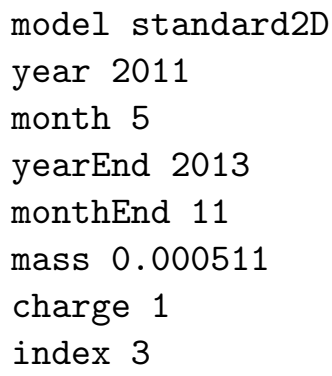

together with the input flux as described above.

The examples in this section show that the simple standard2D model implemented in SOLARPROP is able to describe charge-sign dependent solar modulation. More sophisticated models which better describe the data can be implemented by the user.

\section{Conclusions}

We have introduced a tool called SOLARPROP to simulate charge-sign dependent solar modulation for cosmic rays. This is an advantage to the commonly used force-field approximation, as also drift effects which are charge-sign dependent are taken into account. We have validated the tool against several simple models from the literature, to show that the software works as expected. A more sophisticated two dimensional model has also been implemented. The 


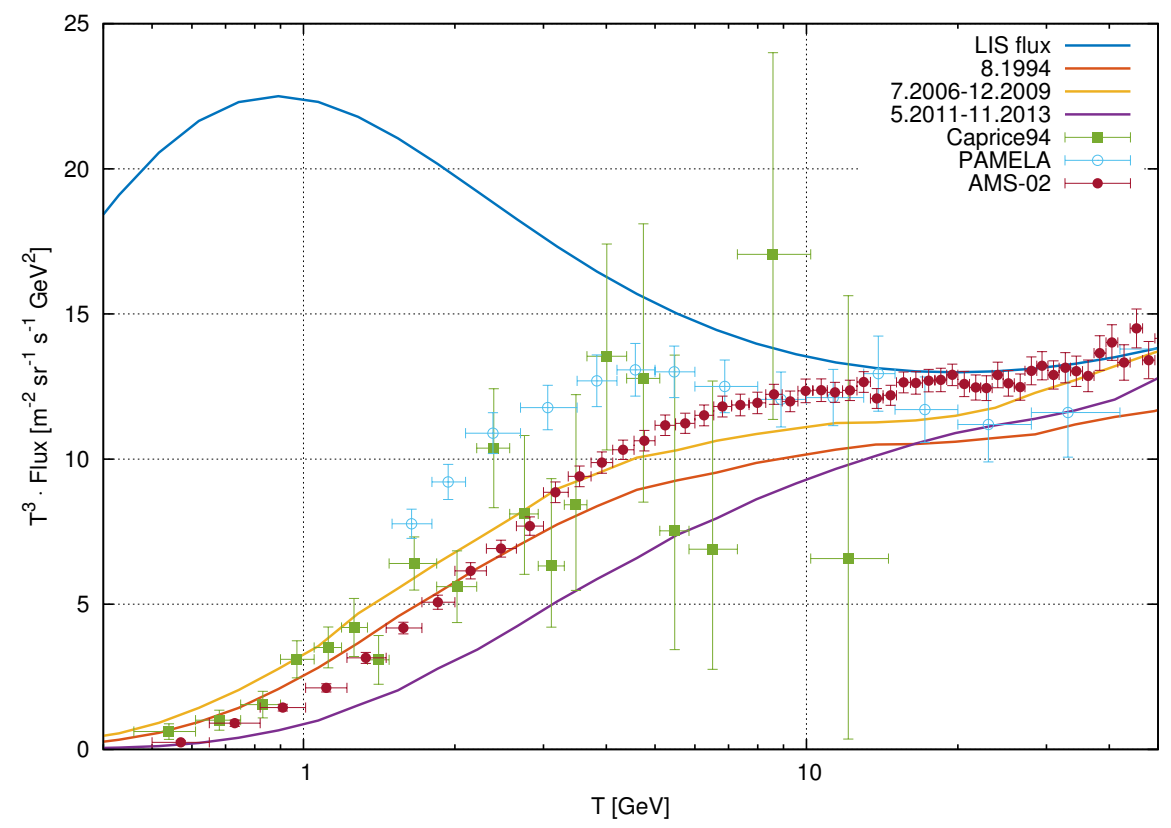

Figure 6: Data for cosmic ray positrons at different solar activities measured by Caprice [46], PAMELA [47] and AMS-02 [48]. The model standard2D is in poor agreement with the data. The question if this indicates that the solar modulation model is too simple or the local interstellar positron flux is different is left for future work.

only required input for this model is a date or a time period. With this information and a local interstellar flux, SOLARPROP calculates the top of the atmosphere flux. The results have been compared with several proton and antiproton data sets from BESS, BESS-Polar and PAMELA and are in a good agreement. The comparison with positron data from PAMELA and AMS-02 is more challenging. We find some tension with the data, which probably show that the implemented model is too simple to account for solar modulation for leptons. On the other hand also the local interstellar flux for positrons is rather uncertain due to its possible primary component.

SOLARPROP has the advantage that it can be easily extended by new models. The implementation of custom models will make it possible to test further models for leptons. This is left for future work. Another advantage of SOLARPROP is its FITS interface. With this interface, output from popular tools for the propagation of cosmic rays in the galaxy like GALPROP and DRAGON can directly used as input for SOLARPROP. In the past, the free Fisk potential in the force-field method was often degenerate with fit parameters from galaxy propagation of cosmic rays like the strength of reacceleration. We hope that SOLARPROP can help to break this degeneracy and will lead in the combination with a detailed study of cosmic ray transport in the galaxy to a smaller propagation uncertainty at $\mathrm{GeV}$ kinetic energies. This is important for indirect dark matter detection with antiprotons and positrons and will hopefully be useful in the near future. 


\section{Acknowledgments}

This work was supported by the SFB-Transregio TR33 "The Dark Universe" (Deutsche Forschungsgemeinschaft) and by the Munich Institute for Astro- and Particle Physics (MIAPP) of the DFG cluster of excellence "Origin and Structure of the Universe".

\section{A Description of the models}

We summarize the different SDEs which are implemented for the different models in SOLARPROP. The rigidity for a particle with momentum $p$, mass $m$ and charge $Z$ is defined as

$$
\mathcal{R}=\frac{p}{|Z|}=\frac{\sqrt{T^{2}+2 T m}}{|Z|}
$$

\section{A.1 One dimensional models}

\section{A.1.1 Model ref1}

The one dimensional model ref $1[10]$ is described by

$$
\begin{aligned}
\Delta r & =\left(-V+\frac{2 \kappa_{r r}}{r}\right) \Delta t+\sqrt{2 \kappa_{r r} \Delta t} d w_{r}, \\
\Delta T & =\frac{2 V}{3 r} \frac{T^{2}+2 T m}{T+m} \Delta t .
\end{aligned}
$$

The solar wind is assumed to be constant $V=400 \mathrm{~km} \mathrm{~s}^{-1}$ and $d w_{j}$ is a Gaussian distribution of random numbers with mean zero and standard deviation of one $N(0,1)$. The radial part of the diffusion tensor is given by

$$
\kappa_{r r}=\kappa_{0} \beta \mathcal{R}=\kappa_{0} \beta \frac{\sqrt{T^{2}+2 T m}}{|Z|} .
$$

$\beta$ is the particle speed which can be linked to the kinetic energy.

\section{A.2 Two dimensional models}

We assume for all models that the heliospheric magnetic field is described by a Parker spiral (see [30] for a recent discussion of different magnetic fields)

$$
\mathbf{B}=\frac{A}{r^{2}}\left(\mathbf{e}_{\mathbf{r}}-\tan \psi \mathbf{e}_{\phi}\right)\left(1-2 H\left(\vartheta-\frac{\pi}{2}\right)\right), \quad B=\frac{|A|}{r^{2}} \sqrt{1+\tan ^{2} \psi}
$$

$A$ is a constant taking care that the magnetic field at the position of the earth matches its measured value $\approx 5 \mathrm{nT}$ and the correct polarity of the solar cycle. We thus have usually $A \approx \pm 3.4 \mathrm{nT} \mathrm{AU}^{2}$. The factor $\pi / 2$ in the Heaviside function is strictly valid only for a flat HCS and has to be corrected otherwise. The spiral angle $\psi$ is defined as

$$
\tan \psi=\Gamma=\frac{\Omega r \sin \vartheta}{V}
$$


where $\Omega=2.866 \cdot 10^{-6} \mathrm{rad} \mathrm{s}^{-1}$ is the average angular rotation of the sun. The radial and angular part of the diffusion tensor can be rewritten 53

$$
\begin{aligned}
& \kappa_{r r}=\kappa_{\|} \cos ^{2} \psi+\kappa_{\perp} \sin ^{2} \psi=\frac{1}{1+\Gamma^{2}}\left(\kappa_{\|}+\kappa_{\perp} \Gamma^{2}\right), \\
& \kappa_{\vartheta \vartheta}=\kappa_{\perp} .
\end{aligned}
$$

For the SDEs we find

$$
\begin{aligned}
\Delta r & =\left(-V-V_{\mathrm{D}, r}-V_{\mathrm{HCS}, r}+\frac{1}{r^{2}} \frac{\partial r^{2} \kappa_{r r}}{\partial r}\right) \Delta t+\sqrt{2 \kappa_{r r} \Delta t} d w_{r}, \\
\Delta \vartheta & =\left(-\frac{V_{\mathrm{D}, \vartheta}}{r}+\frac{1}{r^{2} \sin \vartheta} \frac{\partial \sin \vartheta \kappa_{\vartheta \vartheta}}{\partial \vartheta}\right) \Delta t+\frac{\sqrt{2 \kappa_{\vartheta \vartheta} \Delta t}}{r} d w_{\vartheta}, \\
\Delta T & =\frac{2 V}{3 r} \frac{T^{2}+2 T m}{T+m} \Delta t .
\end{aligned}
$$

\section{A.2.1 Model ref2}

The two dimensional model ref2 26$]$ is described by a $r$ independent diffusion tensor

$$
\begin{aligned}
& \kappa_{\|}=\kappa_{0} \beta \sqrt{\mathcal{R}}=\kappa_{0} \beta \frac{\left(T^{2}+2 T m\right)^{\frac{1}{4}}}{\sqrt{|Z|}}, \\
& \kappa_{\perp}=0.1 \kappa_{\|}
\end{aligned}
$$

which results in

$$
\begin{aligned}
\frac{1}{r^{2}} \frac{\partial r^{2} \kappa_{r r}}{\partial r} & =\frac{2}{r}\left(\kappa_{r r}+\left(\kappa_{\perp}-\kappa_{\|}\right) \frac{\Gamma^{2}}{\left(1+\Gamma^{2}\right)^{2}}\right), \\
\frac{1}{r^{2} \sin \vartheta} \frac{\partial \sin \vartheta \kappa_{\vartheta \vartheta}}{\partial \vartheta} & =\frac{\kappa_{\perp}}{r^{2}} \cot \vartheta .
\end{aligned}
$$

The HCS is assumed to be flat. To avoid a singular drift velocity due to the flat HCS we use a regularization proposed in [54]. In [26] a different approach is used, but our validation shows that the result does not depend on the details of the regularization. The drift velocities are given by

$$
\begin{aligned}
V_{\mathrm{D}, r} & =q \frac{2 \beta r}{3 A} \sqrt{T^{2}+2 T m} \cot \vartheta \frac{\Gamma}{\left(1+\Gamma^{2}\right)^{2}}\left(1-2 H\left(\vartheta-\frac{\pi}{2}\right)\right), \\
V_{\mathrm{D}, \vartheta} & =q \frac{2 \beta r}{3 A} \sqrt{T^{2}+2 T m} \frac{\Gamma\left(2+\Gamma^{2}\right)}{\left(1+\Gamma^{2}\right)^{2}}\left(1-2 H\left(\vartheta-\frac{\pi}{2}\right)\right), \\
V_{\mathrm{HCS}, r} & = \begin{cases}-q \frac{A \beta}{|A|} \frac{\Gamma}{\sqrt{1+\Gamma^{2}}}\left(0.457-0.412 \frac{d}{R_{L}}+0.0915 \frac{d^{2}}{R_{L}^{2}}\right) & , d<2 R_{L} \\
0 & , d \geq 2 R_{L} .\end{cases}
\end{aligned}
$$

where the Larmor radius $R_{L}$ and the distance to the HCS $d$ are given by

$$
\begin{aligned}
R_{L} & =\frac{\mathcal{R}}{B}=\frac{\sqrt{T^{2}+2 T m}}{|Z|} \frac{r^{2}}{|A| \sqrt{1+\Gamma^{2}}}, \\
d & =|r \cos \vartheta| .
\end{aligned}
$$




\section{A.2.2 Model ref3}

Another two dimensional model is the one named ref3 [27]. We find for the diffusion tensor

$$
\begin{aligned}
\kappa_{\|} & = \begin{cases}\kappa_{0 \|} \beta \sqrt{0.4}\left(1+r^{2}\right) & , \mathcal{R}<0.4 \mathrm{GV} \\
\kappa_{0 \|} \beta \sqrt{\mathcal{R}}\left(1+r^{2}\right)=\kappa_{0 \|} \beta \frac{\left(T^{2}+2 T m\right)^{\frac{1}{4}}}{\sqrt{|Z|}}\left(1+r^{2}\right) & , \mathcal{R} \geq 0.4 \mathrm{GV},\end{cases} \\
\kappa_{\perp} & =\kappa_{0 \perp} \beta \mathcal{R} \frac{r^{2}}{\sqrt{1+\Gamma^{2}}}=\kappa_{0 \perp} \beta \frac{\sqrt{T^{2}+2 T m}}{|Z|} \frac{r^{2}}{\sqrt{1+\Gamma^{2}}}
\end{aligned}
$$

which gives us

$$
\begin{aligned}
\frac{1}{r^{2}} \frac{\partial r^{2} \kappa_{r r}}{\partial r} & =\frac{2}{r}\left(\kappa_{r r}+\left(\kappa_{\perp}-\kappa_{\|}\right) \frac{\Gamma^{2}}{\left(1+\Gamma^{2}\right)^{2}}+\frac{r^{2}}{1+r^{2}} \frac{\kappa_{\|}}{1+\Gamma^{2}}+\kappa_{\perp} \frac{\Gamma^{2}\left(2+\Gamma^{2}\right)}{2\left(1+\Gamma^{2}\right)^{2}}\right), \\
\frac{1}{r^{2} \sin \vartheta} \frac{\partial \sin \vartheta \kappa_{\vartheta \vartheta}}{\partial \vartheta} & =\frac{1}{1+\Gamma^{2}} \frac{\kappa_{\perp}}{r^{2}} \cot \vartheta .
\end{aligned}
$$

The model simulates a wavy HCS and introduces a smooth function $f$ and its derivative $f^{\prime}$ to model the HCS drifts. We find

$$
\begin{aligned}
f & =\frac{1}{\alpha_{\mathrm{HCS}}} \arctan \left(\left(1-\frac{2 \vartheta}{\pi}\right) \tan \alpha_{\mathrm{HCS}}\right), \\
f^{\prime} & =-\frac{2}{\pi \alpha_{\mathrm{HCS}}} \frac{\tan \alpha_{\mathrm{HCS}}}{1+\left(1-\frac{2 \vartheta}{\pi}\right)^{2} \tan ^{2} \alpha_{\mathrm{HCS}}}, \\
\alpha_{\mathrm{HCS}} & =\arccos \left(\frac{\pi}{2 \vartheta_{1 / 2}}-1\right) .
\end{aligned}
$$

where $\vartheta_{1 / 2}$ is a reference value for the waviness of the HCS. The drift velocities are

$$
\begin{aligned}
V_{\mathrm{D}, r} & =q \frac{2 \beta r}{3 A} \sqrt{T^{2}+2 T m} \cot \vartheta \frac{\Gamma}{\left(1+\Gamma^{2}\right)^{2}} f, \\
V_{\mathrm{D}, \vartheta} & =q \frac{2 \beta r}{3 A} \sqrt{T^{2}+2 T m} \frac{\Gamma\left(2+\Gamma^{2}\right)}{\left(1+\Gamma^{2}\right)^{2}} f, \\
V_{\mathrm{HCS}, r} & =q \frac{\beta r}{3 A} \sqrt{T^{2}+2 T m} \frac{\Gamma}{1+\Gamma^{2}} f^{\prime} .
\end{aligned}
$$

\section{A.2.3 Model ref4}

The last two dimensional reference model implemented in SOLARPROP is model ref 4 [28]. The diffusion tensor agrees with the one of model ref3. The drift effects are modeled in a different way. The regular part agrees with the one from model ref 2 and is thus given by

$$
\begin{aligned}
& V_{\mathrm{D}, r}=q \frac{2 \beta r}{3 A} \sqrt{T^{2}+2 T m} \cot \vartheta \frac{\Gamma}{\left(1+\Gamma^{2}\right)^{2}}\left(1-2 H\left(\vartheta-\frac{\pi}{2}\right)\right), \\
& V_{\mathrm{D}, \vartheta}=q \frac{2 \beta r}{3 A} \sqrt{T^{2}+2 T m} \frac{\Gamma\left(2+\Gamma^{2}\right)}{\left(1+\Gamma^{2}\right)^{2}}\left(1-2 H\left(\vartheta-\frac{\pi}{2}\right)\right) .
\end{aligned}
$$

The model simulates a wavy HCS with tilt angle $\alpha$ by 55

$$
\vartheta_{\Delta}=\frac{2 \mathcal{R} V}{|A| \Omega \cos \alpha}=\frac{2 \sqrt{T^{2}+2 T m} V}{|A||Z| \Omega \cos \alpha}
$$


and

$$
V_{\mathrm{HCS}, r}= \begin{cases}2 \sin \vartheta \frac{\Gamma}{\sqrt{1+\Gamma^{2}}} \frac{\beta}{6} \frac{\vartheta_{\Delta} \cos \alpha}{\sin \left(\alpha+\vartheta_{\Delta}\right)} & , \frac{\pi}{2}-\alpha-\vartheta_{\Delta}<\vartheta<\frac{\pi}{2}+\alpha+\vartheta_{\Delta} \\ 0 & , \text { else. }\end{cases}
$$

\section{A.2.4 Model standard2D}

The model standard2D incorporates a tilt angle dependent wavy HCS and is tuned against recent cosmic ray data. The diffusion tensor is

$$
\begin{aligned}
\kappa_{\|} & = \begin{cases}\kappa_{0} \frac{|A|}{3 B} \beta \cdot 0.1=\kappa_{0} \beta \frac{1}{30} \frac{r^{2}}{\sqrt{1+\Gamma^{2}}} & , \mathcal{R}<0.1 \mathrm{GV} \\
\kappa_{0} \frac{|A|}{3 B} \beta \mathcal{R}=\kappa_{0} \beta \frac{\sqrt{T^{2}+2 T m}}{3|Z|} \frac{r^{2}}{\sqrt{1+\Gamma^{2}}} & , \mathcal{R} \geq 0.1 \mathrm{GV},\end{cases} \\
\kappa_{\perp} & =0.02 \kappa_{\|}
\end{aligned}
$$

which results in

$$
\begin{aligned}
\frac{1}{r^{2}} \frac{\partial r^{2} \kappa_{r r}}{\partial r} & =\frac{2}{r}\left(\kappa_{r r}+\left(\kappa_{\perp}-\kappa_{\|}\right) \frac{\Gamma^{2}}{\left(1+\Gamma^{2}\right)^{2}}+\kappa_{r r} \frac{\left(2+\Gamma^{2}\right)}{2\left(1+\Gamma^{2}\right)}\right), \\
\frac{1}{r^{2} \sin \vartheta} \frac{\partial \sin \vartheta \kappa_{\vartheta \vartheta}}{\partial \vartheta} & =\frac{1}{1+\Gamma^{2}} \frac{\kappa_{\perp}}{r^{2}} \cot \vartheta .
\end{aligned}
$$

The normalization of the diffusion tensor $\kappa_{0}$ is time dependent (see also equation 7). For the drift effects the model is inspired by [12] and thus similar to model ref 3 . The only difference is that $\vartheta_{1 / 2}$ is related to the tilt angle of the current sheet $\alpha$ by 28$]$

$$
\vartheta_{1 / 2}=\frac{\pi}{2}-\frac{1}{2} \sin \left(\alpha+\frac{2 R_{L}}{r}\right)=\frac{\pi}{2}-\frac{1}{2} \sin \left(\alpha+\frac{\sqrt{T^{2}+2 T m}}{|Z|} \frac{2 r}{|A| \sqrt{1+\Gamma^{2}}}\right) .
$$

\section{References}

[1] D. Maurin, F. Donato, R. Taillet, and P. Salati, Astrophys. J. 555 (2001), 585-596, [astro-ph/0101231].

[2] GALPROP, http://galprop.stanford.edu.

[3] A. W. Strong and I. V. Moskalenko, Astrophys. J. 509 (1998), 212-228, [astro$\mathrm{ph} / 9807150]$.

[4] DRAGON, http://dragonproject.org.

[5] C. Evoli, D. Gaggero, D. Grasso, and L. Maccione, JCAP 0810 (2008), 018, [0807.4730].

[6] E. Parker, Planetary and Space Science 13 (1965), no. 1, 9 - 49.

[7] L. J. Gleeson and W. I. Axford, Astrophys. J. 154 (1968), 1011.

[8] H. Gast and S. Schael, Proceedings of the 31st ICRC Lodz (2009).

[9] I. Cholis, D. Hooper, and T. Linden, (2015), 1511.01507.

[10] Y. Yamada, S. Yanagita, and T. Yoshida, Geophys. Res. Letters 25 (1998), 2353-2356. 
[11] M. Zhang, Astrophys.J. 513 (1999), 409-420.

[12] P. Bobik, G. Boella, M. Boschini, C. Consolandi, S. Della Torre, et al., Astrophys.J. 745 (2012), 132, [1110.4315].

[13] R. D. Strauss, M. S. Potgieter, I. Büsching, and A. Kopp, Astrophys. J. 735 (2011), 83.

[14] C. Pei, J. W. Bieber, R. A. Burger, and J. Clem, Journal of Geophysical Research (Space Physics) 115 (2010), no. A14, 12107.

[15] K. Alanko-Huotari, I. G. Usoskin, K. Mursula, and G. A. Kovaltsov, Journal of Geophysical Research (Space Physics) 112 (2007), 8101.

[16] M. S. Potgieter, E. E. Vos, M. Boezio, N. De Simone, V. Di Felice, and V. Formato, Solar Phys. 289 (2014), 391, [1302.1284].

[17] SOLARPROP, http://www.th.physik.uni-bonn.de/nilles/people/kappl/.

[18] R. Kappl and M. W. Winkler, Phys. Rev. D85 (2012), 123522, [1110.4376].

[19] J. R. Jokipii and E. N. Parker, Astrophys.J. 160 (1970), 735.

[20] C. W. Gardiner, (1989), Handbook of Stochastic Methods, Springer Verlag, Berlin.

[21] A. Kopp, I. Büsching, R. D. Strauss, and M. S. Potgieter, Computer Physics Communications 183 (2012), 530-542.

[22] S. Della Torre et al., (2015), 1511.00541.

[23] GALPROP Manual, http://galprop.stanford.edu/download/manuals/galprop_v54.pdf.

[24] WSO, http://wso.stanford.edu/Tilts.html.

[25] I. G. Usoskin et al., http://cosmicrays.oulu.fi/phi/Phi_mon.txt.

[26] J. R. Jokipii and D. A. Kopriva, Astrophys.J. 234 (1979), 384-392.

[27] M. S. Potgieter and H. Moraal, Astrophys.J. 294 (1985), 425-440.

[28] R. A. Burger and M. S. Potgieter, Astrophys.J. 339 (1989), 501-511.

[29] O. Adriani et al., Astrophys. J. 765 (2013), 91, [1301.4108].

[30] J. L. Raath, M. S. Potgieter, R. D. Strauss, and A. Kopp, (2015), 1506.07305.

[31] J. T. Hoeksema, Space Science Reviews 72 (1995), 137-148.

[32] SILSO World Data Center, International Sunspot Number Monthly Bulletin and online catalogue http://www.sidc.be/silso/.

[33] Neutron monitors of the Bartol Research Institute, http://neutronm.bartol.udel.edu.

[34] I. G. Usoskin, K. Alanko-Huotari, G. A. Kovaltsov, and K. Mursula, Journal of Geophysical Research (Space Physics) 110 (2005), no. A9, 12108. 
[35] I. G. Usoskin, G. A. Bazilevskaya, and G. A. Kovaltsov, Journal of Geophysical Research (Space Physics) 116 (2011), 2104.

[36] E. C. Stone, A. C. Cummings, F. B. McDonald, B. C. Heikkila, N. Lal, and W. R. Webber, Science 341 (2013), 150-153.

[37] R. A. Burger, M. S. Potgieter, and B. Heber, Journal of Geophysical Research 105 (2000), 27447-27456.

[38] BESS, S. Orito et al., Phys. Rev. Lett. 84 (2000), 1078-1081, [astro-ph/9906426].

[39] S. Haino, K. Abe, H. Fuke, T. Maeno, Y. Makida, et al., International Cosmic Ray Conference 3 (2005), 13.

[40] Y. Shikaze et al., Astropart. Phys. 28 (2007), 154-167, [astro-ph/0611388].

[41] K. Abe et al., Phys. Lett. B670 (2008), 103-108, [0805.1754].

[42] K. Abe et al., Phys. Rev. Lett. 108 (2012), 051102, [1107.6000].

[43] K. Abe et al., (2015), 1506.01267.

[44] R. Kappl, A. Reinert, and M. W. Winkler, JCAP 1510 (2015), no. 10, 034, [1506.04145].

[45] R. Kappl and M. W. Winkler, JCAP 1409 (2014), 051, [1408.0299].

[46] G. Barbiellini, G. Basini, R. Bellotti, M. Bocciolini, M. Boezio, et al., Astronomy and Astrophysics 309 (1996), L15-L18.

[47] PAMELA, O. Adriani et al., Phys. Rev. Lett. 111 (2013), 081102, [1308.0133].

[48] AMS, M. Aguilar et al., Phys. Rev. Lett. 113 (2014), 121102.

[49] L. Maccione, Phys.Rev.Lett. 110 (2013), no. 8, 081101, [1211.6905].

[50] M. Di Mauro, F. Donato, N. Fornengo, and A. Vittino, (2015), 1507.07001.

[51] M. S. Potgieter, E. E. Vos, R. Munini, M. Boezio, and V. Di Felice, Astrophys. J. 810 (2015), no. 2, 141.

[52] O. Adriani et al., Astrophys. J. 810 (2015), no. 2, 142.

[53] R. A. Burger, T. P. J. Krüger, M. Hitge, and N. E. Engelbrecht, Astrophys. J. 674 (2008), 511-519.

[54] R. A. Burger, H. Moraal, and G. M. Webb, Astrophysics and Space Science 116 (1985), $107-129$.

[55] R. A. Burger and M. Hattingh, Astrophysics and Space Science 230 (1995), 375-382. 